\title{
The Mechanical Properties of Defective Graphyne
}

\author{
Shuting Lei ${ }^{1}$, Qiang Cao ${ }^{1, *}$, Xiao Geng ${ }^{1}$, Yang Yang ${ }^{1}$, Sheng Liu ${ }^{1}$ and Qing Peng ${ }^{2, *(\mathbb{D}}$ \\ 1 The Institute of Technological Sciences, Wuhan University, Wuhan 430072, China; \\ shutinglei@whu.edu.cn (S.L.); xiaogeng@whu.edu.cn (X.G.); yy1163685316@163.com (Y.Y.); \\ Victor_liu63@126.com (S.L.) \\ 2 Nuclear Engineering and Radiological Sciences, University of Michigan, Ann Arbor, MI 48109, USA \\ * Correspondence: caoqiang@whu.edu.cn (Q.C.); qpeng.org@gmail.com (Q.P); Tel.: +86-027-68776698 (Q.C.); \\ $+1-158-279-6669$ (Q.P.)
}

Received: 24 November 2018; Accepted: 9 December 2018; Published: 12 December 2018

\begin{abstract}
Graphyne is a two-dimensional carbon allotrope with superior one-dimensional electronic properties to the "wonder material" graphene. In this study, via molecular dynamics simulations, we investigated the mechanical properties of $\alpha_{-}, \beta_{-}, \delta_{-}$, and $\gamma$-graphynes with various type of point defects and cracks with regard to their promising applications in carbon-based electronic devices. The Young's modulus and the tensile strength of the four kinds of graphyne were remarkably high, though still lower than graphene. Their Young's moduli were insensitive to various types of point defects, in contrast to the tensile strength. When a crack slit was present, both the Young's modulus and tensile strength dropped significantly. Furthermore, the Young's modulus was hardly affected by the strain rate, indicating potential applications in some contexts where the strain rate is unstable, such as the installation of membranes.
\end{abstract}

Keywords: graphyne; mechanical properties; stress-strain; Young's modulus; tensile strength; strain rate

\section{Introduction}

Since the fantastic properties of graphene were discovered in 2004 [1], there have been extensive research studies studying its unique properties and explore its allotropes with broad application prospects [2-6]. As an appealing member of the graphene allotrope family, graphyne is a one-atom-thick sheet of carbon atoms with both $\mathrm{sp}^{2}$ carbon bonds and sp hybridized bonds. With the change in the proportion of $\mathrm{sp}^{2}$ carbon bonds replaced by the acetylenic linkages, various types of graphynes with different geometries can be formed [7,8]. Four typical graphynes- $\alpha-, \beta-, \delta-$ and $\gamma$-graphynes-are shown in Figure $1 \mathrm{a}-\mathrm{d}$, respectively. The percentages of the acetylenic linkages were $100 \%, 66.67 \%, 41.67 \%$, and $33.33 \%$ for the $\alpha-, \beta-, \delta$ - and $\gamma$-graphynes, respectively. After it was predicted by Baughman et al. [9] that graphyne would have a much lower crystalline state formation energy and stability at high-temperatures, several attempts were made to explore graphyne's properties and potential for promising applications [10-13]. Among these properties, the mechanical properties play a very important role, and many studies have been carried out to investigate the stiffness, Young's modulus, Poisson's ratio, etc., of various graphynes, especially for the four typical graphynes, using molecular dynamic (MD) simulations and ab initio calculations since 2011 [14-16].

Among these four typical graphynes, the $\gamma$-graphyne has been studied several times. The superior mechanical robustness and tolerance of delta graphyne membranes, verified by biaxial tensile tests, can reduce the possible deformations effectively during the installation of the membrane in hydrogen purification [17]. Later, further investigation into the mechanical and electronic properties of the $\gamma$-graphyne using the first-principles calculations was carried out. The results showed that the in-plane stiffness decreased with an increasing number of acetylenic linkages [18]. The influence of vacancies 
on the delta graphyne was investigated by Ajori et al [19]. It is worth noting that the arrangement of vacancy was also related to the final results [20]. In addition to the theoretical research, researchers have been working on synthesizing graphyne. Thus far, $\gamma$-graphyne has been successfully synthesized among these four graphynes [21], indicating its broad application in catalysis, gas separation and energy-related fields. Compared to the extensive studies on the properties of perfect graphyne, the mechanical properties of graphyne with a few point defects or cracks have received much less attention and have not yet been investigated systematically. It is commonly acknowledged that defects are widespread in real materials and play an essential role in the materials' properties. Therefore, it is of great importance and practical use to study defective graphyne [22-24]. Herein, we investigated the Young's moduli and tensile strengths of four types of graphyne with point defects or cracks. The influences of the strain rate on the mechanical properties was examined in tensile tests using molecular dynamic (MD) simulations.
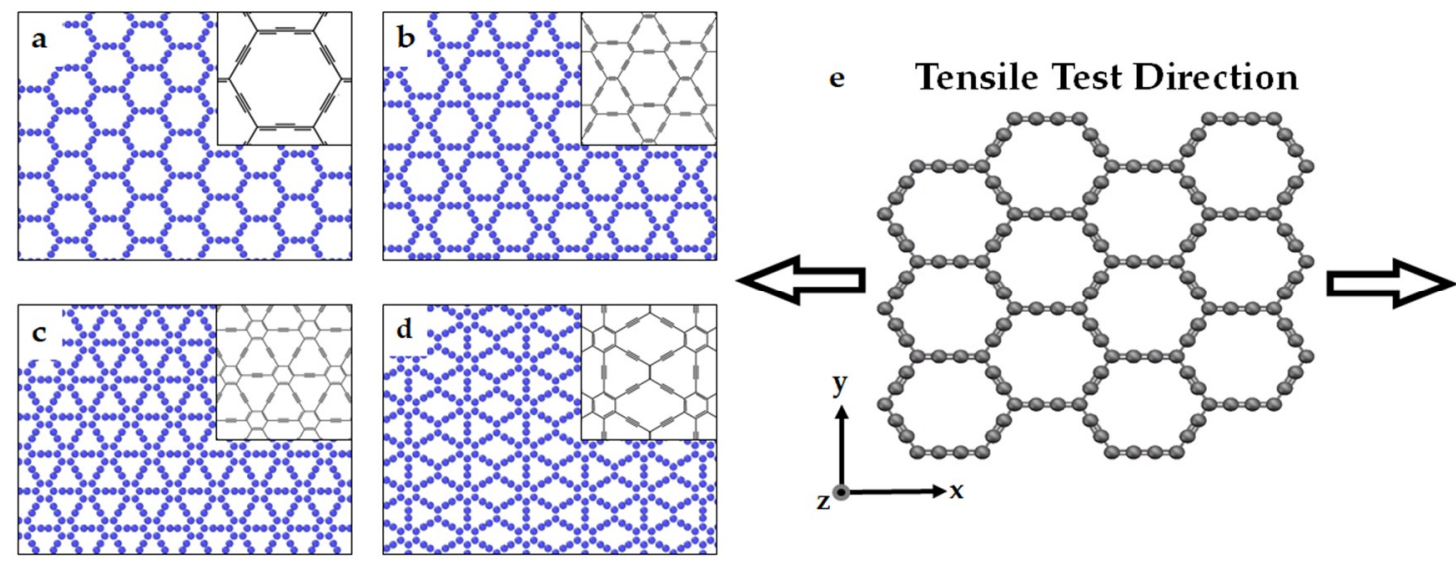

Figure 1. The structure of four types of graphyne and the schematic diagram of the tensile test: (a) $\alpha$ graphyne; (b) $\beta$-graphyne; (c) $\delta$-graphyne; (d) $\gamma$-graphyne; (e) schematic diagram of the tensile test.

\section{Materials and Methods}

The MD simulations were carried out using the Large-Scale Atomic/Molecular Massively Parallel Simulator (LAMMPS) package [25]. The equilibrium atomic structures and tensile test setup are shown in Figure 1. For the four perfect graphyne models, we used $12 \times 10 \times 3 \mathrm{~nm}^{3}(2400$ atoms), $11 \times 11 \times 3$ $\mathrm{nm}^{3}$ (3024 atoms), $11 \times 11 \times 3 \mathrm{~nm}^{3}$ (3456 atoms) and $11 \times 12 \times 3 \mathrm{~nm}^{3}$ (3804 atoms) simulation boxes for the $\alpha-, \beta-, \delta$ - and $\gamma$-graphynes, respectively. For the four types of graphyne with point defects, we investigated 7 types of point defects—one point defect (1-1 type), two adjacent point defects (2-a type), two point defects not far apart (2-c type), two point defects far apart (2-f type), three adjacent point defects (3-a type), three point defects not far apart (3-c type), three point defects far apart (3-f type)-as shown in Figure 2a-g. For the four types of graphyne with a crack, the crack was perpendicular to the y direction, as shown in Figure $2 \mathrm{~h}$. The boundary conditions set were periodic, periodic, and fixed in the $\mathrm{x}, \mathrm{y}$, and $\mathrm{z}$ directions, respectively.

The force-field that describes the interactions between carbon atoms is the adaptive intermolecular reactive bond order (AIREBO) [26], which allows for covalent bond breaking and forming, enabling an accurate description of the interactions between atoms under extreme conditions, including fracture. The simulation temperature was maintained at $300 \mathrm{~K}$ using a Nose-Hoover thermostat $[27,28]$. The simulation time step was 0.0005 ps. Before tensile loading, the structure was fully relaxed using the isothermal-isobaric ensemble (constant temperature and constant pressure ensemble, or NPT ensemble). After equilibrium, uniaxial tensile loading was applied along the $x$ direction, which was like the armchair direction in graphene. The strain rate was set at $0.5 \times 10^{9} \mathrm{~s}^{-1}$. In this study, all the strain, stress, Young's moduli, and tensile strengths were specified to the $\mathrm{x}$ direction. 

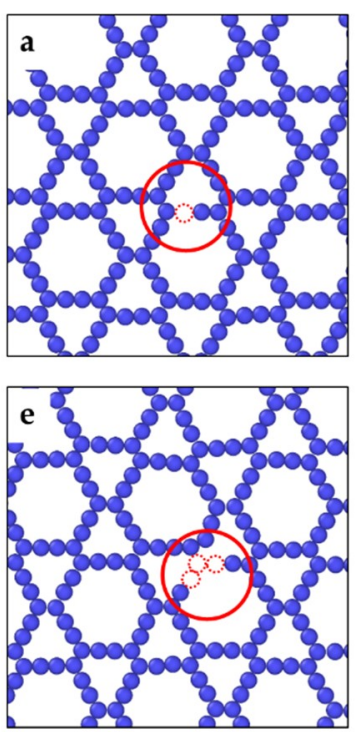
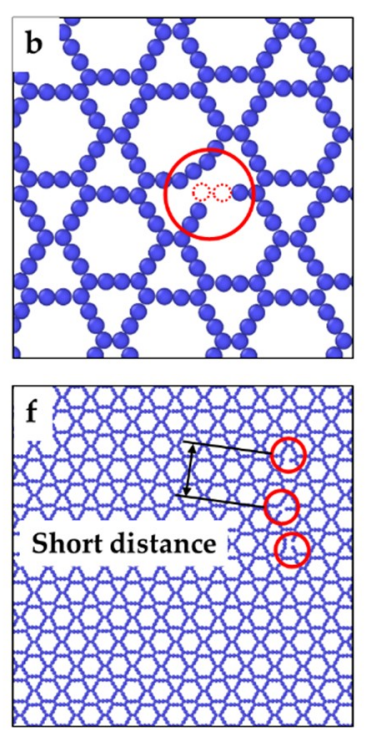
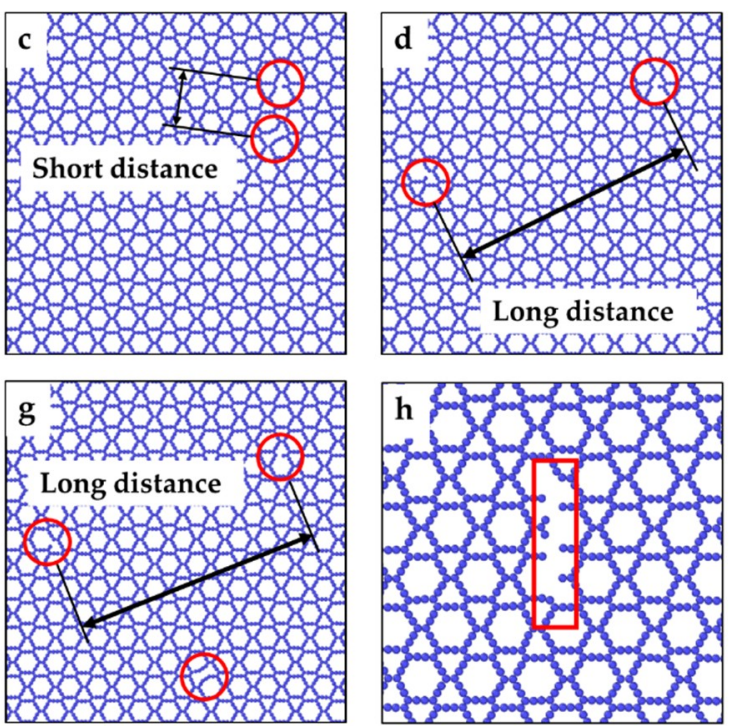

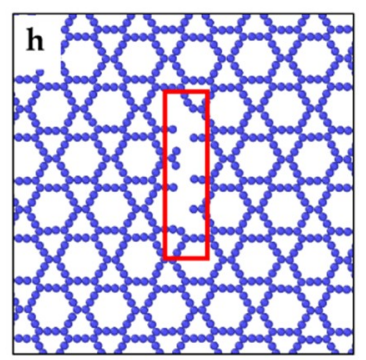

Figure 2. Various types of graphyne with defects and graphyne with a crack. (a) One point defect (1-1 type); (b) Two adjacent point defects (2-a type); (c) Two point defects not far apart (2-c type); (d) Two point defects far apart (2-f type); (e) Three adjacent point defects (3-a type); (f) Three point defects not far apart (3-c type); (g) Three point defects far apart (3-f type); (h) Graphyne with a crack.

\section{Results and Discussion}

\subsection{Strain-Stress Relationship}

\subsubsection{Perfect Graphyne}

Before the tensile tests, we first tested different sizes and length-width ratios of graphyne supercells to ensure that the simulation sizes in our models were large enough to minimize the size effects. We tested 11 supercells, including eight supercells generated by a unit cell $(1 \times 1)$ expanding its length in the $x$ direction with multiples from one to eight, and another three supercells of the size $2 \times 2,3 \times 3$ and $4 \times 4$. Each unit cell covered an approximate square with a side length of about $11 \mathrm{~nm}$. The results showed that the average Young's modulus was $177.56 \mathrm{GPa}$ with a standard deviation of $0.2788 \mathrm{GPa}$, indicating that the Young's moduli of supercells with different sizes and length-width ratios were almost the same. This meant that the mechanical properties from the simulations in the following models were not related to the sizes and length-width ratios, and the following test results for the $1 \times 1$ cells should be reliable.

We then performed molecular dynamics simulations on the perfect graphyne. The results are shown in Figure 3a,b. The Young's moduli were obtained from Hooke's law $\sigma=E \varepsilon$, when the stress $\sigma$ and strain $\varepsilon$ show a linear relationship in the first stage of the stress-strain curve. The thickness of the structure was considered $0.334 \mathrm{~nm}$ in order to provide the area of a cross-section vertical to the strain direction to calculate the stress. The tensile strength was defined as the maximum stress in the tensile test, that is, the ordinate of the highest point in the stress-strain curve. This value reflects the upper strength limit of the system.

As Figure 3a shows, the Young's moduli of the $\alpha-, \beta-, \delta$ - and $\gamma$-graphynes were $178.5 \mathrm{GPa}$, 339.7 GPa, 476.9 GPa, and 538.5 GPa, respectively. Previous researchers also obtained the Young's moduli of $\delta$-graphyne and $\gamma$-graphyne using DFT (Density Functional Theory) and MD calculations, respectively. The Young's modulus of the $\delta$-graphyne was $162 \mathrm{~N} / \mathrm{m}$ (if they used a thickness of 0.334 , as in our study, the Young's modulus would have been $485 \mathrm{GPa}$ ), and the Young's modulus of the $\gamma$-graphyne was $510 \mathrm{GPa}[19,29]$. Our results $(476.9 \mathrm{GPa}$ and $538 \mathrm{GPa})$ match well with theirs. The percentages of the acetylenic linkages were $100 \%, 66.67 \%, 41.67 \%$, and $33.33 \%$ for the $\alpha-, \beta-, \delta$ - and $\gamma$-graphyne, respectively [18]. With an increasing number of acetylenic linkages in place of $\mathrm{sp}^{2}$ carbon 
bonds, the Young's modulus of graphyne declined sharply. In other words, the acetylenic linkages can significantly reduce the ability of graphyne to resist deformation.
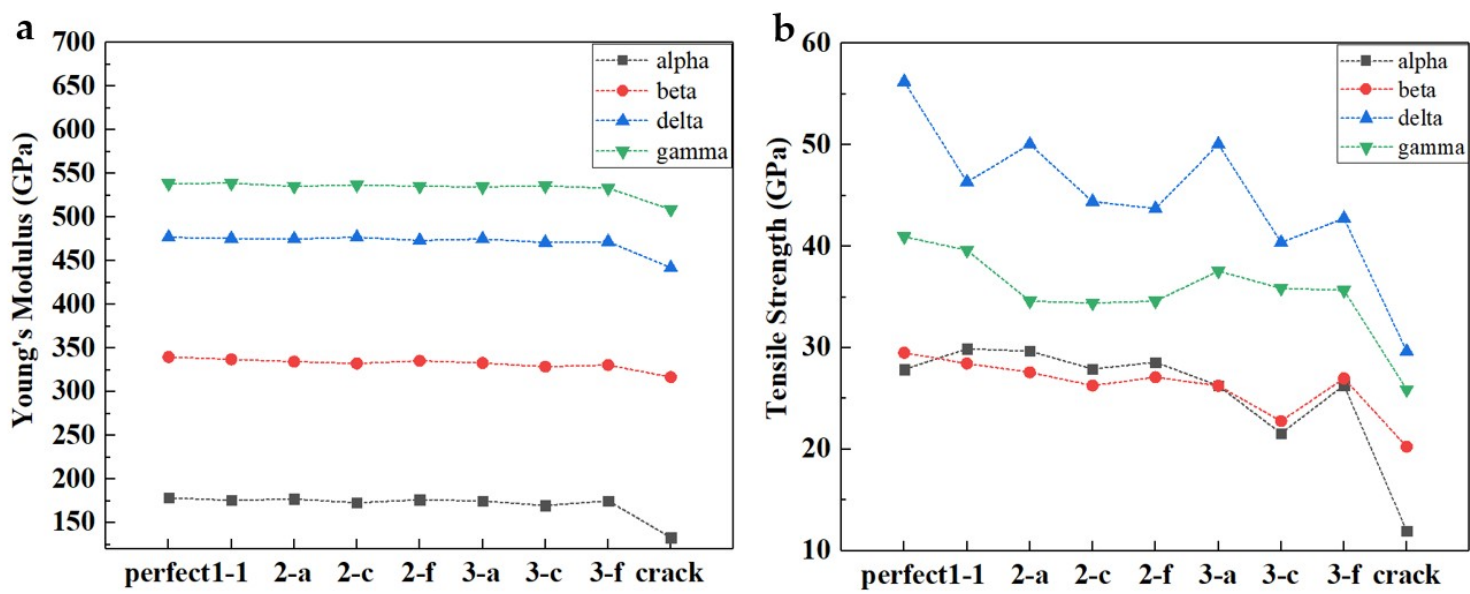

Figure 3. (a) The Young's moduli of perfect graphyne and graphyne with vacancies or a crack, (b) The tensile strengths of perfect graphyne and graphyne with vacancies or a crack.

\subsubsection{Graphynes with Vacancies}

When addressing the practical applications of this material, we considered the influence of point defects, because the vacancies might have a significant influence on the mechanical properties of graphyne [30]. Here, we concentrated on seven kinds of vacancies (1-1, 2-a, 2-c, 2-f, 3-a, 3-c, 3-f) and studied their effects on the Young's modulus and fracture strength, separately. As shown in Figure 3a, the Young's modulus almost remained constant. The standard deviations of the Young's modulus for the four types of perfect graphyne and graphyne with defects were 2.67 GPa, 1.98 GPa, 2.48 GPa, and $5.11 \mathrm{GPa}$, respectively, indicating that these four types of graphyne still had a good ability to resist deformation even with a few vacancies. These phenomena show the potential of graphyne to maintain its mechanical properties when it has a few vacancies.

With regard to the tensile strength, the influence of various kinds of vacancies was complicated. It seems that the influence of different types of vacancies depended more on the structure and properties of the graphyne. In other words, the same type of vacancies had a very different effect on different materials. However, there are still some phenomena worthy of our attention. For the $\beta-, \delta-$, and $\gamma$-graphynes, the tensile strength was reduced without exception in the presence of vacancies. However, with the one- or two-point vacancies, the tensile strength of the $\alpha$-graphyne was a little larger than the perfect graphyne especially; this may be because the structure of the $\alpha$-graphyne was not stable enough. Further research might be required to explain the phenomenon. Meanwhile, for the $\alpha$-graphyne, we noted that the impact of the other various vacancies was not large, except for the 3-c type. In other kinds of graphyne, we also observed a distinct drop of tensile strength in the presence of 3-c-type point vacancies. One possible explanation for this is that three-point vacancies that are relatively close to each other are more likely to expand into a crack (Figure 4), while the impact of the other types of two- or three-point vacancies is just equivalent to the impact of a corresponding single vacancy in the same type of graphyne. 


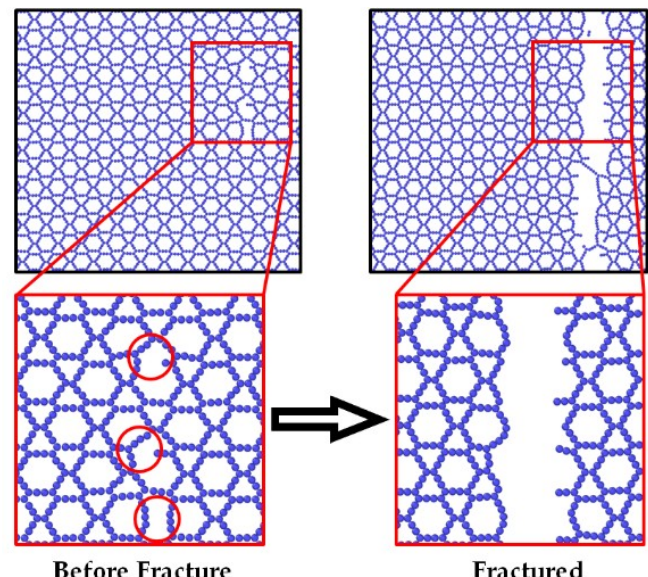

Figure 4. Three-point vacancies not far apart (3-c type) are likely to expand into a crack.

\subsubsection{Graphyne with Crack}

When researching a new material, we should not only focus on the perfect material. It is worth also paying more attention to the material with cracks. The useful strength of large-area graphyne with engineering relevance is usually determined by its tensile strength, rather than the intrinsic strength that governs a uniform breaking of atomic bonds in perfect graphyne [20]. Thus, it is necessary for us to attach importance to graphyne with cracks. Before we determined the size of the cracks for the testing, we first explored how large the crack can be before it affects the properties of the graphyne. We did a crack atom test on $\beta$-graphyne. We generated a crack in each of three different $\beta$-graphyne sheets by removing the atoms in three corresponding regions, including $0.2 \times 2.0 \times 3.0 \mathrm{~nm}^{3}$ (12 atoms), $0.4 \times 4.0 \times 3.0 \mathrm{~nm}^{3}\left(44\right.$ atoms) and $0.4 \times 9.0 \times 3.0 \mathrm{~nm}^{3}$ (99 atoms). These removed atoms accounted for $0.4 \%, 1.5 \%$, and $3.3 \%$ of the total number of atoms, respectively. It can be seen in Figure 5 that the stress-strain curve of the $\beta$-graphyne with the 12-atom crack was close to that of the perfect graphyne before it yielded. The graphyne with 44-atom or 99-atom cracks had mechanical properties far from those of the perfect graphene. Based on this, we hold the view that it was reasonable that the number of atoms in the crack should not exceed $1 \%$ of the total number of atoms.

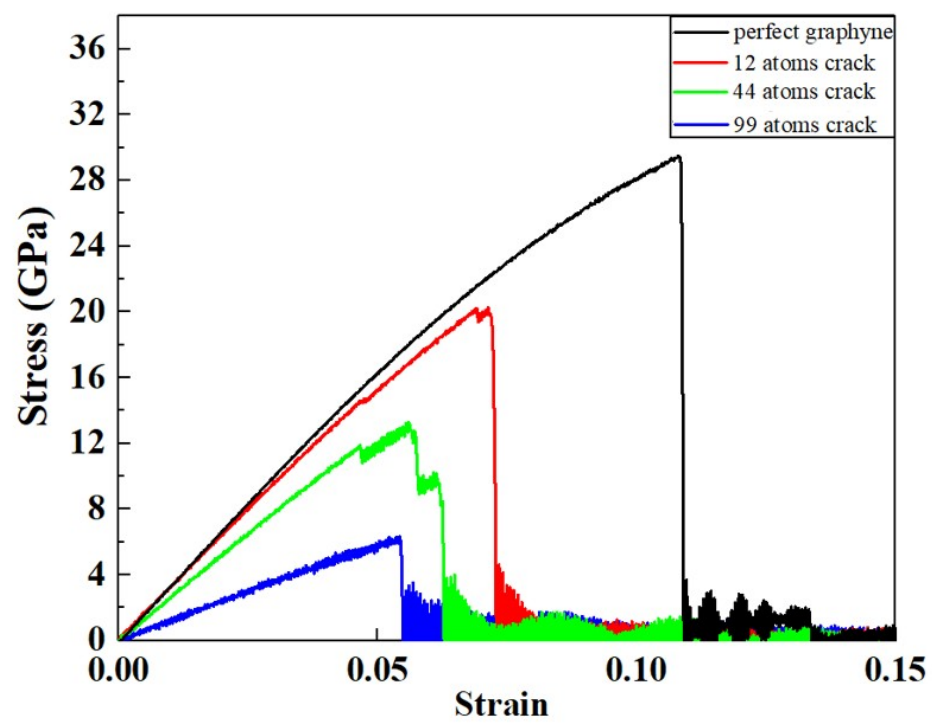

Figure 5. Stress-strain curves of four types of graphyne under tensile loading.

As a result of this investigation, we separately generated $0.2 \times 2.0 \times 3.0 \mathrm{~nm}^{3}$ ( 6 atoms), $0.2 \times 2.0$ $\times 3.0 \mathrm{~nm}^{3}$ (12 atoms), $0.1 \times 2.0 \times 3.0 \mathrm{~nm}^{3}\left(11\right.$ atoms) and $0.1 \times 2.0 \times 3.0 \mathrm{~nm}^{3}$ (11 atoms) cracks for 
$\alpha-, \beta-, \delta-$ and $\gamma$-graphynes, respectively. The proportion of missing atoms was maintained under $1 \%$ to make the results reliable. After the system was fully relaxed, we carried out the tensile test and obtained the stress-strain curve.

From the comparison in Table 1 and Figure 3a,b, we can observe the strong influence of the crack on the mechanical properties of the graphyne in terms of both the Young's modulus and the tensile strength. Taking $\alpha$-graphyne as an example, we can see that the Young's modulus was reduced to $132.85 \mathrm{GPa}$, a drop of $25.56 \%$ from that of the perfect system, and the tensile strength decreased to $11.95 \mathrm{GPa}$, a sharp decline of $57.13 \%$. This data implies that the structure of $\alpha$-graphyne is not stable and is very sensitive to cracks. The Young's modulus and tensile strength of the other three graphynes also suffered a drop of various degrees. Among them, it is notable that the change in the Young's modulus and tensile strength of $\beta$-graphyne was relatively small. This might be due to the unique structure of $\beta$-graphyne. In Figure 1 , there is a triangular structure, which might make $\beta$-graphyne more stable and less sensitive to cracks. This discovery might explain $\beta$-graphyne's outstanding performance when a crack is generated.

Table 1. Comparison of the Young's modulus and tensile strength of four types of perfect graphyne and graphyne with a crack.

\begin{tabular}{|c|c|c|c|c|c|c|}
\hline Type & $\begin{array}{l}\text { Crack Young's } \\
\text { Modulus/GPa }\end{array}$ & $\begin{array}{l}\text { Perfect Young's } \\
\text { Modulus/GPa }\end{array}$ & $\Delta \mathrm{Y} / \mathrm{GPa}$ & $\begin{array}{l}\text { Crack Tensile } \\
\text { Strength/GPa }\end{array}$ & $\begin{array}{c}\text { Perfect Tensile } \\
\text { Strength/GPa }\end{array}$ & $\Delta \mathrm{f} / \mathrm{GPa}$ \\
\hline$\alpha$-graphyne & 132.85 & 178.47 & 45.62 & 11.95 & 27.88 & 15.93 \\
\hline$\beta$-graphyne & 316.86 & 339.72 & 22.86 & 20.25 & 29.50 & 9.25 \\
\hline$\delta$-graphyne & 441.98 & 476.88 & 34.90 & 29.64 & 65.22 & 26.58 \\
\hline$\gamma$-graphyne & 508.83 & 538.53 & 29.71 & 25.88 & 40.95 & 15.07 \\
\hline
\end{tabular}

\subsection{Strain Rate Effect}

Since the material needs time to react to the applied mechanical loading, the strain rate also plays an important role in the final results. Here, we set four different strain rates- $0.5 \times 10^{9} \mathrm{~s}^{-1}$, $1 \times 10^{9} \mathrm{~s}^{-1}, 4 \times 10^{9} \mathrm{~s}^{-1}$ and $10 \times 10^{9} \mathrm{~s}^{-1}$-for four types of graphyne with a crack. Unless otherwise stated, the settings for the other conditions are consistent with those mentioned above. Figure 6 depicts the test results.
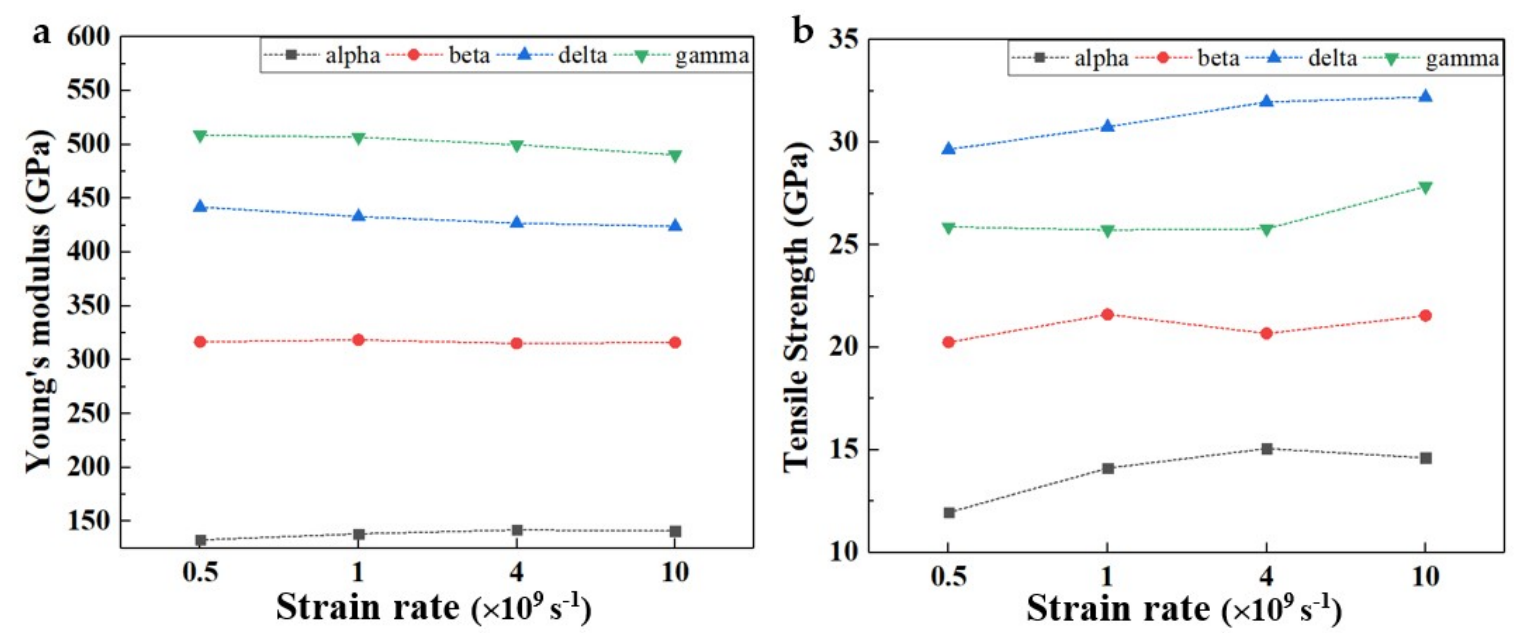

Figure 6. (a) Young's modulus and (b) tensile strength for four types of graphyne under different strain rates.

For the Young's modulus, the strain rate has little influence on the final results. All the ratios of the standard deviation to the average were under the $3 \%$ obtained from the statistics, indicating the slight effect that the strain rate had on the Young's modulus from another point of view. This result is in good agreement with the results of Zhang's study [31], which investigated the effect of the strain 
rate on the $\gamma$-graphyne. With the increase of the strain rate, the tensile strength also showed an upward trend. When the strain rate is low, atoms have more time to perform thermal fluctuations, and are therefore more likely to overcome the energy barrier and cause the bonds to break. This may be the reason that there is a dependent relationship between the strain rate and the fracture strength.

\section{Conclusions}

In summary, we investigated the effect of seven types of point defects and one type of crack on the four types of graphyne under uniaxial tension, and the strain rate effect on the mechanical properties using MD simulations. We found that the Young's moduli of the defective graphynes almost remained constant with the perfect graphyne and maintained a high level $(17.50 \%, 33.31 \%, 46.75 \%$, $52.80 \%$ that of graphene). The ratio of the Young's modulus reduction was positively correlated with the proportion of $\mathrm{sp}^{2}$ carbon bonds replaced by the acetylenic linkages. When a 3-c type of point defect exists, the tensile strength drops distinctly. Moreover, the existence of a crack can result in a significant decrease in Young's modulus and tensile strength, while the change in Young's modulus and tensile strength of the $\beta$-graphyne with a crack was relatively small. Furthermore, the Young's modulus was insensitive to the strain rate, and the tensile strength increased slightly when the strain rate increased.

Author Contributions: Conceptualization, S.L. (Shuting Lei); Formal analysis, S.L. (Shuting Lei), Q.C. and X.G.; Funding acquisition, Q.C. and S.L. (Sheng Liu); Investigation, S.L. (Shuting Lei) and Y.Y.; Methodology, S.L. (Shuting Lei); Visualization, X.G. and S.L. (Shuting Lei); Writing-original draft, S.L. (Shuting Lei), Q.C., and Y.Y.; Writing-review \& editing, X.G. and Q.P.; Supervision, Q.C., S.L. (Sheng Liu), and Q.P.

Funding: We acknowledge the calculation support of the Supercomputing Center of Wuhan University and the financial support from the National Natural Science Foundation of China (No. 51727901).

Conflicts of Interest: The authors declare no conflict of interest.

\section{References}

1. Novoselov, K.S.; Geim, A.K.; Morozov, S.V.; Jiang, D.; Zhang, Y.; Dubonos, S.V.; Grigorieva, I.V.; Firsov, A.A. Electric field effect in atomically thin carbon films. Science 2004, 306, 666-669. [CrossRef] [PubMed]

2. Schedin, F.; Geim, A.K.; Morozov, S.V.; Hill, E.W.; Blake, P.; Katsnelson, M.I.; Novoselov, K.S. Detection of individual gas molecules adsorbed on graphene. Nat. Mater. 2007, 6, 652-655. [CrossRef] [PubMed]

3. Lee, C.; Wei, X.; Kysar, J.W.; Hone, J. Measurement of the elastic properties and intrinsic strength of monolayer graphene. Science 2008, 321, 385-388. [CrossRef] [PubMed]

4. Binghui, D.; Jie, H.; Hanxing, Z.; Sheng, L.; Emily, L.; Yunfeng, S.; Qing, P. The normal-auxeticity mechanical phase transition in graphene. 2D Mater. 2017, 4, 021020. [CrossRef]

5. Peng, Q.; Han, L.; Lian, J.; Wen, X.; Liu, S.; Chen, Z.; Koratkar, N.; De, S. Mechanical degradation of graphene by epoxidation: Insights from first-principles calculations. Phys. Chem. Chem. Phys. 2015, 17, 19484-19490. [CrossRef]

6. Peng, Q.; Liang, C.; Ji, W.; De, S. A theoretical analysis of the effect of the hydrogenation of graphene to graphane on its mechanical properties. Phys. Chem. Chem. Phys. 2013, 15, 2003-2011. [CrossRef]

7. Coluci, V.R.; Braga, S.F.; Legoas, S.B.; Galvão, D.S.; Baughman, R.H. New families of carbon nanotubes based on graphyne motifs. Nanotechnology 2004, 15, S142. [CrossRef]

8. Peng, Q.; Dearden, A.K.; Crean, J.; Han, L.; Liu, S.; Wen, X.; De, S. New materials graphyne, graphdiyne, graphone, and graphane: Review of properties, synthesis, and application in nanotechnology. Nanotechnol. Sci. Appl. 2014, 7, 1-29. [CrossRef]

9. Baughman, R.H.; Eckhardt, H.; Kertesz, M. Structure-property predictions for new planar forms of carbon: Layered phases containing sp2 and sp atoms. J. Chem. Phys. 1987, 87, 6687-6699. [CrossRef]

10. Cranford, S.W.; Buehler, M.J. Selective hydrogen purification through graphdiyne under ambient temperature and pressure. Nanoscale 2012, 4, 4587-4593. [CrossRef]

11. Falcao, E.H.; Wudl, F. Carbon allotropes: Beyond graphite and diamond. J. Chem. Technol. Biotechnol. 2007, 82, 524-531. [CrossRef]

12. Qian, X.; Ning, Z.; Li, Y.; Liu, H.; Ouyang, C.; Chen, Q.; Li, Y. Construction of graphdiyne nanowires with high-conductivity and mobility. Dalton Trans. 2012, 41, 730-733. [CrossRef] [PubMed] 
13. Peng, Q.; Zamiri, A.R.; Ji, W.; De, S. Elastic properties of hybrid graphene/boron nitride monolayer. Acta Mech. 2012, 223, 2591-2596. [CrossRef]

14. Enyashin, A.N.; Ivanovskii, A.L. Graphene allotropes. Phys. Status Solidi (b) 2011, 248, 1879-1883. [CrossRef]

15. Hirsch, A. The era of carbon allotropes. Nat. Mater. 2010, 9, 868-871. [CrossRef] [PubMed]

16. Yang, Y.; Xu, X. Mechanical properties of graphyne and its family-A molecular dynamics investigation. Comput. Mater. Sci. 2012, 61, 83-88. [CrossRef]

17. Lin, S.; Buehler, M.J. Mechanics and molecular filtration performance of graphyne nanoweb membranes for selective water purification. Nanoscale 2013, 5, 11801-11807. [CrossRef] [PubMed]

18. Zhang, Y.Y.; Pei, Q.X.; Wang, C.M. Mechanical properties of graphynes under tension: A molecular dynamics study. Appl. Phys. Lett. 2012, 101, 081909. [CrossRef]

19. Ajori, S.; Ansari, R.; Mirnezhad, M. Mechanical properties of defective $\gamma$-graphyne using molecular dynamics simulations. Mater. Sci. Eng. A 2013, 561, 34-39. [CrossRef]

20. Zhang, P.; Ma, L.; Fan, F.; Zeng, Z.; Peng, C.; Loya, P.E.; Liu, Z.; Gong, Y.; Zhang, J.; Zhang, X.; et al. Fracture toughness of graphene. Nat. Commun. 2014, 5, 3782. [CrossRef] [PubMed]

21. Gao, X.; Zhu, Y.; Yi, D.; Zhou, J.; Zhang, S.; Yin, C.; Ding, F.; Zhang, S.; Yi, X.; Wang, J.; et al. Ultrathin graphdiyne film on graphene through solution-phase van der waals epitaxy. Sci. Adv. 2018, 4. [CrossRef] [PubMed]

22. Amorim, R.G.; Fazzio, A.; Antonelli, A.; Novaes, F.D.; da Silva, A.J.R. Divacancies in graphene and carbon nanotubes. Nano Lett. 2007, 7, 2459-2462. [CrossRef] [PubMed]

23. Cao, Q.; Geng, X.; Wang, H.; Wang, P.; Liu, A.; Lan, Y.; Peng, Q. A review of current development of graphene mechanics. Crystals 2018, 8, 357. [CrossRef]

24. López-Polín, G.; Gómez-Navarro, C.; Parente, V.; Guinea, F.; Katsnelson, M.I.; Pérez-Murano, F.; Gómez-Herrero, J. Increasing the elastic modulus of graphene by controlled defect creation. Nat. Phys. 2014, 11, 26-31. [CrossRef]

25. Plimpton, S. Fast parallel algorithms for short-range molecular dynamics. J. Comput. Phys. 1995, 117, 1-19. [CrossRef]

26. Pearlman, D.A.; Case, D.A.; Caldwell, J.W.; Ross, W.S.; Cheatham, T.E.; DeBolt, S.; Ferguson, D.; Seibel, G.; Kollman, P. Amber, a package of computer programs for applying molecular mechanics, normal mode analysis, molecular dynamics and free energy calculations to simulate the structural and energetic properties of molecules. Comput. Phys. Commun. 1995, 91, 1-41. [CrossRef]

27. Nosé, S. A unified formulation of the constant temperature molecular dynamics methods. J. Chem. Phys. 1984, 81, 511-519. [CrossRef]

28. Hoover, W.G. Canonical dynamics: Equilibrium phase-space distributions. Phys. Rev. A 1985, 31, $1695-1697$. [CrossRef]

29. Peng, Q.; Ji, W.; De, S. Mechanical properties of graphyne monolayers: A first-principles study. Phys. Chem. Chem. Phys. 2012, 14, 13385-13391. [CrossRef] [PubMed]

30. PENG, Q.; CREAN, J.; DEARDEN, A.K.; Huang, C.; Wen, X.; Bordas, S.P.A.; De, S. Defect engineering of 2d monatomic-layer materials. Mod. Phys. Lett. B 2013, 27, 1330017. [CrossRef]

31. Zhang, Y.; Pei, Q.; Mai, Y.-W.; Gu, Y. Temperature and strain-rate dependent fracture strength of graphynes. J. Phys. D Appl. Phys. 2014, 47, 425301. [CrossRef]

(C) 2018 by the authors. Licensee MDPI, Basel, Switzerland. This article is an open access article distributed under the terms and conditions of the Creative Commons Attribution (CC BY) license (http://creativecommons.org/licenses/by/4.0/). 